\title{
ANÁLISE DE SENTIMENTOS DOS ALBERGUES DE SANTA CATARINA ATRAVÉS DOS COMENTÁRIOS NO SITE TRIPADVISOR
}

\author{
Luciana Ordine Araújo ${ }^{1}$
}

\begin{abstract}
Resumo: O presente artigo busca fazer uma relação entre o que é exposto nas mídias sociais e sites de relacionamentos com clientes e qual o impacto que estas informações pessoais, dadas pelos viajantes que já se hospedaram nesse tipo de estabelecimento, trazem para os albergues/hostels localizados no Estado de Santa Catarina. Para que este estudo fosse realizado, foi efetuada uma busca em seis sites na internet, onde foi possível buscar informações sobre os albergues existentes em Santa Catarina, e opiniões dos viajantes. Após analisar os sites, e verificar que aquele que possuía maior número de comentários foi o Tripdavisor, ele foi utilizado para realizar a análise de sentimentos com a ferramenta Tagul e o R. Através destas ferramentas de análise de sentimentos foi possível emitir um parecer sobre como estas informações podem influenciar na reputação dos locais e se as informações são relevantes ou apenas opiniões isoladas.
\end{abstract}

Palavras-Chave: Hospedagem, Albergues, Santa Catarina, Análise de Sentimentos, Mineração de Dados, Rede Social.

\begin{abstract}
This paper seeks to make a link between what is displayed in social media and customer relationships websites and what impact this personal information, given by travelers who have already stayed in this type of establishment, brings to the hostels located in the Santa Catarina State. For this study to be carried out, a search was made in six websites on the Internet, where it was possible to find information about existing hostels in Santa Catarina, and traveler's opinions. After analyzing these sites and verify that Tripadvisor was the site with the highest number of comments, it was used to perform sentiment analysis with tool's Tagul and R. Through these tools of sentiment analysis it was possible to express an opinion on how this information can influence the reputation of the sites and whether the information is relevant or only isolated opinions.
\end{abstract}

Keywords: Hosting, Hostels, Santa Catarina, Sentiment Analysis, Data Mining, Social Networking.

\section{INTRODUÇÃO}

A ideia central deste artigo é analisar de que maneira as informações dadas na internet pelos viajantes, podem impactar na reputação dos albergues/hostels de Santa Catarina.

O objetivo desta proposta seria verificar quais são as ferramentas de análises de sentimento existentes e qual delas seria mais interessante utilizar para descobrir o sentimento 
das pessoas em relação a uma avaliação feita em um site e qual o grau de confiabilidade destas informações.

O processo acontece da seguinte forma: os viajantes dão suas opiniões a respeito do seu período de hospedagem tendo uma perspectiva real dos locais, como são as acomodações, banheiros e área comum, além da localização e atendimento e cordialidade e através destes sites ou redes sociais emite uma nota sobre a sua hospedagem no local, de horrível até excelente.

E utilizando estes dados através da análise de sentimentos será possível verificar se os dados informados no Tripadvisor podem ser usados como uma maneira de melhorar a reputação dos albergues/hostels.

\section{REFERENCIAL TEÓRICO}

\subsection{História Dos Albergues/Hostels}

Historicamente, os albergues eram casas onde era oferecida comida e bebida mediante pagamento. Durante o Império Romano, este tipo de hospedagem foi bastante difundido. Em 26 de agosto de 1909, o professor alemão Richard Schirmann idealizou a criação dos Albergues depois de refugiar-se ao longo de uma estrada após uma tempestade. Ele dedicava parte de seu tempo a criar programas de convivência para seus alunos. De atividades pedagógicas, passou a organizar grupos com os jovens para realizar pequenas viagens de estudos. Foi assim que o professor Schirmann descobriu a possibilidade de criar uma alternativa para acomodar os alunos, que não fosse apenas a pernoite em hospedarias (FEDERAÇÃO, 2005). O primeiro albergue começou a funcionar três anos mais tarde, 1912, em um castelo em Altena, na Alemanha, monumento histórico restaurado e que funciona até hoje. Ele tinha o objetivo de proporcionar hospedagem barata para jovens e promover a integração entre eles. No final da década de 20, o alberguismo se difundiu por toda a Europa. Nesta época, houve um verdadeiro boom de albergues, que se espalharam por todo continente. Em 1927, foram criados albergues na Suíça e na Polônia; em 1929, na Holanda; em 1930, na Inglaterra, Noruega e França; e, em 1931, na Irlanda, Bélgica e Escócia.

Em 1932, foi criada a International Youth Hostel Federation - IYHF (Federação Internacional de Albergues da Juventude). E, a partir desse momento, o movimento tornou-se, de fato, internacional, com a entrada de diversos países à Federação (HOSTELLING, 2005). Durante a Segunda Guerra Mundial, o movimento permaneceu estagnado e muitos albergues foram destruídos. Em 1945, ao final do conflito, os mesmos começaram a ser restaurados e o movimento passou a ser considerado como uma forma de reintegração da juventude europeia. 


\section{ANÁLISE DE SENTIMENTOS DOS ALBERGUES DE SANTA CATARINA ATRAVÉS DOS COMENTÁRIOS NO SITE...}

Só em 1934 o alberguismo chegou ao continente americano, mais especificamente nos Estados Unidos. A Argentina (1956) e o Uruguai (1958) foram os primeiros países sulamericanos a fazer parte do movimento. Ambos, inclusive, trabalharam pela expansão da rede na América e abriram as portas para o ingresso de Chile, Peru, Colômbia, Bolívia, Equador, Costa Rica, El Salvador e Brasil.

Os Albergues chegaram ao Brasil em 1961, através do casal de educadores cariocas Joaquim e Ione Trotta, que trouxeram a ideia para o País depois de terem visitado um albergue na França, em 1956. O primeiro Albergue brasileiro recebeu o nome de "Residência Ramos" e foi instalado no bairro de Ramos, no Rio de Janeiro. Permaneceu aberto de 1965 a 1973. Neste mesmo período, funcionavam no estado de São Paulo dois Albergues, um na Capital e outro em Campos do Jordão, que foram fechados pelo Governo Militar sob a alegação de reunir jovens universitários (FEDERAÇÃO, 2005). Em 1971, foi fundada a Federação Brasileira de Albergues da Juventude (FBAJ), com sede no Rio de Janeiro, e em 1984 a Associação Paulista de Albergues da Juventude (APAJ), que durante a gestão de Franco Montoro à frente do Governo de São Paulo recebeu apoio significativo, inclusive com a doação de quatro imóveis - um em Campos do Jordão, um em Pindamonhangaba (ambos usados anteriormente por funcionários da Estrada de Ferro Campos do Jordão), um imóvel em Ubatuba e uma casa no Parque Estadual do Jaraguá, que foi habitada pelo bandeirante Afonso Sardinha.

No início de 1986, João Dória Júnior assumiu a presidência da EMBRATUR e incentivou o trabalho dos Albergues, tornando-os conhecidos em todo o Brasil. EM 1996, a FBAJ adotou o sistema de classificação dos Albergues da Juventude em três categorias: Muito Bom, Bom e Regular. A FBAJ estabelece metas e diretrizes de desenvolvimento para suas afiliadas e mantém contatos com as demais federações do mundo. Segundo informações obtidas com a Associação de Albergues da Juventude do Estado do Rio de Janeiro (ALBERJ), esse sistema é baseado no sistema de classificação da ABIH, mas com as devidas adaptações para os Albergues da Juventude. Dependendo da pontuação, foram excluídos da Federação os Albergues da Juventude que não atingiram a pontuação mínima para albergues de categoria regular. Atualmente, a FBAJ não credencia nenhum albergue que obtenha a classificação Regular (somente Bom ou Muito Bom), mas um Albergue da Juventude pode ser rebaixado a essa categoria caso deixe de atender algumas normas ou deixe de fazer manutenções regularmente. Do período de criação até os dias atuais, o movimento alberguista cresceu se profissionalizou e obteve lugar de destaque no panorama internacional.

69 Revista Competitividade e Sustentabilidade - ComSus, Paraná, v. 3, n. 2, p. 67-81, Jul /Dez. 2016. 


\subsection{Definições De Albergues}

Os albergues estão inseridos na categoria de estabelecimentos extra-hoteleiros e podem ser descritos, de acordo com Beni (2003), como estabelecimentos comerciais de hospedagem, subvencionados ou não, que possuem instalações e serviços básicos para atender demanda específica de alojamento de segmentos sociais com recursos financeiros reduzidos.

A OMT (2001) reconhece que, apesar da referência do alojamento turístico internacional ser o hotel, há o crescimento de outras formas de alojamentos em alternativa a este meio tradicional, sob a denominação de extra-hoteleiros ou não hoteleiros. Dentro dessa categoria encontram-se: acampamentos, albergues, apartamentos, chalés e vilas, colônias de férias ("resorts"), balneários, propriedades rurais ("cottages"), "bed\&breakfast" e outros que, apesar de agrupados na mesma categoria, são marcados por diferenças relevantes. As definições abaixo seguem a classificação da Organização Mundial de Turismo (2001, p. 90):

Os acampamentos são uma modalidade de hospedagem na qual se presta serviço de alojamento mediante divisão ou parcelamento de um terreno para uso privativo, para instalação do sistema próprio de alojamento do cliente, ou seja, barracas ou trailers. Neste local, as instalações em comuns são compartilhadas, como: sanitários, tanques de lavar roupa, piscina, salões de jogos, etc. com finalidade de proporcionar ao usuário o desfrute ao ar livre com algumas comodidades.

Os "Self-catering" englobam estabelecimentos que oferecem instalações, equipamentos e utensílios suficientes para desenvolver atividades domésticas necessárias por um determinado tempo. Exemplos destas acomodações são apartamentos, vilas e chalés.

A principal diferença entre hotéis e albergues é que os primeiros oferecem apenas quartos privativos. São estruturas que se limitam a oferecer aos hóspedes um lugar cômodo para dormir e, de acordo com o preço, também oferece outras facilidades. Já os albergues oferecem leitos em dormitórios que podem ter de quatro a mais de 20 camas. Os dormitórios podem ser pequenos ou grandes, só para mulheres, só para homens ou mistos. Também oferecem quartos privativos. Contudo, a proposta de hospedagem propiciada é muito diferente se comparada à de um hotel comum.

Os albergues possuem uma atmosfera alegre, jovial, na qual os hóspedes buscam uma maior interação entre eles, o ambiente é mais descontraído, pois como a maioria são mochileiros, que possuem um estilo mais despojado, sem preocupações com luxos. 


\section{ANÁLISE DE SENTIMENTOS DOS ALBERGUES DE SANTA CATARINA ATRAVÉS DOS COMENTÁRIOS NO SITE...}

\subsection{Gestão da Informação e Conceitos}

\subsubsection{Informação}

No que diz respeito à informação, é possível entender que vários dados associados com um significado formam uma informação.

De acordo com Tarapanoff (2006, p.23) a informação pode ser considerada: um fator determinante para a melhoria de processos, produtos e serviços, tendo valor estratégico em organizações. A ideia da informação como ferramenta estratégica evoluiu depois que a gestão da informação mudou de seu foco inicial de gestão de documentos e dados, para recursos informacionais, mostrando resultados em relação à eficiência operacional, evitando desperdício e automatizando processos.

\subsubsection{Gestão da Informação}

Para Tarapanoff (2006, p. 22) o "principal objetivo da gestão da informação é identificar e potencializar recursos informacionais de uma organização ou empresa e sua capacidade de informação, ensinando-a aprender e adaptar-se a mudanças ambientais.”.

\subsubsection{Mineração de Dados}

Mineração de Dados é uma área multidisciplinar que incorpora técnicas utilizadas em diversas áreas como Inteligência Artificial, especialmente Aprendizado de Máquina (AM) (Monard \& Baranauskas 2003), Base de Dados e Estatística. Por isso, as técnicas utilizadas em MD não devem ser vistas como substitutas de outras formas de análises (por VENIA 398 exemplos, OLAP), mas como praticas para melhorar os resultados das explorações feitas com as ferramentas atualmente utilizadas. O foco central de Mineração de Dados e o de como transformar dados armazenados em conhecimento, expresso em termos de formalismos de representação, tal como regras e relações entre dados. Existe conhecimento que pode ser extraído diretamente de dados sem o uso de qualquer técnica, entretanto, existe também muito conhecimento que está de certa forma "embutida" na Base de Dados, na forma de relações existentes entre itens de dados que, para ser extraído, e necessário o desenvolvimento de técnicas especiais. Assim na próxima seção são definidos os conceitos básicos dados, informação e conhecimento para em seguida abordar o processo de Mineração de Dados como um todo.

71 Revista Competitividade e Sustentabilidade - ComSus, Paraná, v. 3, n. 2, p. 67-81, Jul /Dez. 2016. 


\subsubsection{Mineração de Dados em Mídias Sociais}

O conceito de mineração de conteúdos em mídias sociais ou social media mining (SMM) é em sua essência um processo de mineração web (web mining) e envolve o desenvolvimento de ferramentas e aplicação de técnicas para coletar, monitorar, analisar, resumir e visualizar conteúdos de mídias sociais (Zeng; Chen; Lusch; Li, 2010; Sakaki; Okazaki; Matsuo, 2010; Zafarani; Abbasi; Liu, 2014). Crooks, Croitoru, Stefanidis e Radzikowski (2013), apresentam que a Mineração em Mídias Sociais consiste em três etapas gerais, a saber: i) extração de dados dos provedores e servidores de mídias sociais através das interfaces de programação de aplicativos (API); ii) análise, integração e armazenamento de dados; e iii) análise dos dados para extrair informações de interesse.

Crooks et al. (2013) ressaltam que existem diversas ferramentas e sites que executam parte destes processos de monitoramento em mídias sociais, mas que a grande maioria são limitadas em relação ao tratamento de grandes conjuntos de dados, adicionar contexto ao conteúdo coletado ou para realizar análise detalhada, exigindo assim, o desenvolvimento de sistemas personalizados para executar as etapas de descoberta de conhecimento a partir de conteúdos de mídias sociais. Laine e Fruhwirth (2010) acrescentam que essas ferramentas de monitoramento de mídias sociais são serviços de software oferecidos através da Internet para filtrar e analisar o conteúdo textual produzido por e na mídia social. Estas ferramentas buscam e encontram os conteúdos baseados nas palavras-chave definidas pelos usuários e incorporam diversas outras funcionalidades como análise de volume, fonte, autor, palavra-chave, região, sentimento e oferecem opções para análises, gerar relatórios, gráficos, entre outros.

\subsection{Mineração de Opinião ou Análise de Sentimentos}

\subsubsection{Conceitos}

A análise de sentimentos ou mineração de dados é um campo multidisciplinar que envolve áreas como ciência da computação, linguística, estatística e até psicologia. De maneira geral tem por objetivo analisar o sentimento expresso pelo autor de um texto em seu conteúdo, quando a intenção é identificar o sentimento expresso em uma classe específica, como positivo ou negativo é tida como uma tarefa de classificação.

Em termos técnicos Bing Liu (2012, p.1) afirma que: Análise de sentimento, também chamada de mineração de opinião, é o campo de estudo que analisa as opiniões, sentimentos, avaliações, atitudes e emoções das pessoas em relação às entidades, tais como, produtos, serviços, organizações, pessoas, problemas, eventos, temas, e seus atributos. 


\section{ANÁLISE DE SENTIMENTOS DOS ALBERGUES DE SANTA CATARINA ATRAVÉS DOS COMENTÁRIOS NO SITE...}

Liu (2012) enfatiza que se um documento opinativo avalia mais de uma entidade então os sentimentos sobre as entidades podem ser diferentes, ou seja, o titular da opinião pode ter opiniões positivas em relação a algumas entidades e negativas sobre outras. $\mathrm{O}$ autor afirma ainda, que quando isto ocorre não é recomendado que se faça uma classificação a nível de documento. (Liu, 2012, p.30). No sentido de explicar melhor o funcionamento desta etapa Liu (2012) explica que:

A classificação do sentimento é geralmente formulada em duas classes para a classificação do problema, positiva e negativa. Os dados de treino e teste utilizados para a classificação são geralmente sobre avaliação de produtos. Alguns comentários on-line tem pontuação atribuída por usuários, por exemplo, 1-5 estrelas, a classificação positiva ou negativa pode ser determinada usando as notas. Ou seja, uma avaliação com 4 ou 5 estrelas é classificada como positiva, já uma avaliação com 1 ou 2 estrelas é considerada negativa. A maioria dos trabalhos de pesquisa não utiliza a classe neutra, o que deixa a tarefa consideravelmente mais fácil, porém também é possível utilizar esta classe, por exemplo, a atribuição de 3 estrelas pode ser classificada como neutra. (Liu, 2012, p.31).

A análise de sentimentos encontra utilização em diversos setores do mercado consumidor, tais como avaliação de produtos, descoberta de atitudes e suas tendências de consumidores para o fortalecimento de campanhas de marketing, encontrar opiniões acerca de tópicos em alta ou também avaliar lmes. Pang e Lee apresentam alguns empregos da análise de sentimentos, como: Sites de avaliação; Subcomponente de sistemas e Inteligência de mercado e inteligência governamental.

\subsubsection{Como a análise de sentimentos é realizada:}

Pode ser feita de diversas formas como:

- Algoritmos: Utiliza algoritmos matemáticos complexos chamados de "processamento de linguagem natural” para descobrir quais palavras são consideradas positivas e quais são negativas. 
-Tratamento Estatístico: Quanto aos classificadores estatísticos, Pang, Lee \& Vaithyanathan (2002) referem três métodos bastante utilizados para a classificação de textos: Naïve Bayes, Maximum Entropy e Support Vector Machines.

-Naiive Bayes: O modelo de AS, é frequentemente utilizado o classificador de Bayes, pela facilidade de implementação e também pelos resultados positivos que se obtém nas tarefas de classificação. Como classificador, é considerado um dos mais eficientes em questões relacionadas com processamento e precisão na classificação de novas amostras. Thomas Bayes desenvolveu-o em meados do século XVIII e é normalmente chamado de fórmula de probabilidade condicional de um determinado evento, muito utilizado em Machine Learning (Junior, 2008).

- Support Vector Machine (SVM): é um algoritmo de classificação, tenta encontrar um hiperplano ou conjunto de hiperplanos numa dimensão alta (ou infinita) de forma a obter uma boa separação entre as classes das instâncias. Esta separação pode ser definida como a maior distância entre os elementos mais próximos de classes distintas, apesar de que, em geral, quanto maior essa distância, menor o poder de generalização.

\subsubsection{Modelos}

O modelo que será utilizado nesta pesquisa serão as nuvens de palavras. As nuvens de palavras, ou tag clouds. As nuvens são uma forma de representação da importância de diferentes termos em um corpus - um texto específico, um conjunto de textos, ou mesmo um conjunto de conjuntos de texto (a internet, e sua manta de palavras espalhadas). A tecnologia por trás das nuvens é muito delicada, e envolve fórmulas matemáticas para calcular o peso relativo de cada termo com base em sua frequência e daí derivar sua importância na representação, por meio de tamanho de fonte, cor, etc.

As nuvens de palavras possibilitam inúmeras aplicações. A mais comum é a representação da importância de um conjunto de metadados em sites e blogs (como a que temos no nosso próprio blog). Entretanto, há também aplicações importantes em pesquisas nas áreas de linguística e ciência da informação.

\subsubsection{Ferramentas}

Entre as ferramentas disponíveis, foi efetuada uma pesquisa e após uma análise dos dados, foram tabeladas as principais conforme abaixo: 


\section{ANÁLISE DE SENTIMENTOS DOS ALBERGUES DE SANTA CATARINA ATRAVÉS DOS COMENTÁRIOS NO SITE...}

\begin{tabular}{|c|c|c|c|c|c|c|}
\hline \multicolumn{2}{|c|}{ Recursos } & Gratuita & \multirow{2}{*}{$\begin{array}{l}\text { Paga } \\
\text { Não } \\
\end{array}$} & \multirow{2}{*}{$\begin{array}{l}\text { Disponibilidade Web } \\
\text { Sim }\end{array}$} & \multirow{2}{*}{\begin{tabular}{|l} 
Instalaçao \\
Desktop
\end{tabular}} & \multirow{2}{*}{\begin{tabular}{|l} 
O Que Faz \\
Análise De Sentimentos No Twitter \\
\end{tabular}} \\
\hline 1 & Market Place & Sim & & & & \\
\hline 2 & Meaning Cloud & Não & Sim & Não & Sim & Analise De Palavras Do Excel \\
\hline 3 & Mention & Sim & Não & Sim & Não & $\begin{array}{l}\begin{array}{l}\text { Análise De Palavras Em Vários } \\
\text { Sites }\end{array} \\
\end{array}$ \\
\hline 4 & $\mathrm{R}$ & Sim & Não & Não & Sim & Nuvem De Palavras \\
\hline 5 & Phyton & Sim & Não & Não & Sim & Análise De Palavras \\
\hline 6 & Seekr & Não & Sim & Sim & Não & $\begin{array}{l}\text { Análise De Sentimentos Das Redes } \\
\text { Sociais }\end{array}$ \\
\hline 7 & Sentiment 140 & Sim & Não & Sim & Não & Análise De Sentimentos No Twitter \\
\hline 8 & Semantria & Não & Sim & Não & Sim & Analise De Palavras Do Excel \\
\hline 9 & Social Mention & Sim & Não & Sim & Não & $\begin{array}{l}\text { Análise De Palavras Em Blogs, E } \\
\text { Outros Sites } \\
\end{array}$ \\
\hline 10 & Tagul & Sim & Não & Sim & Não & Nuvem De Palavras \\
\hline 11 & Text2data & Não & Sim & Não & Sim & Análise De Sentimentos No Twitter \\
\hline 12 & Tweet Feel & Sim & Não & Sim & Não & Análise De Sentimentos No Twitter \\
\hline 13 & Twendz & Sim & Não & Sim & Não & Análise De Sentimentos No Twitter \\
\hline 14 & Twitrratr & Sim & Não & Sim & Não & Análise De Sentimentos No Twitter \\
\hline 15 & Umigon & Sim & Não & Sim & Não & Análise De Sentimentos No Twitter \\
\hline 16 & Weka & Sim & Não & Não & Sim & Analise De Palavras Do Excel \\
\hline 17 & \#Opinionmining & Sim & Não & Sim & Não & $\begin{array}{l}\text { Análise De Palavras, Gera Gráficos } \\
\text { E Nuvem De Palavras }\end{array}$ \\
\hline 18 & Vismkt & $\mathrm{NaO}$ & Sim & $\mathrm{NaO}$ & Sim & 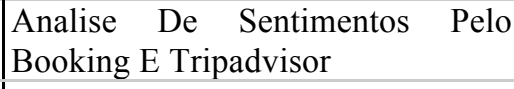 \\
\hline 19 & Protege & Sim & Não & Não & Sim & Analise De Palavras \\
\hline 20 & Swasi & Não & Sim & Não & Sim & 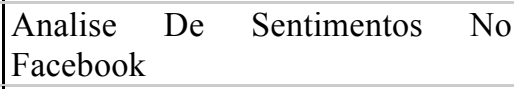 \\
\hline 21 & Opiniontracker & Não & Sim & Sim & Não & $\begin{array}{l}\text { Análise De Sentimentos Nas Redes } \\
\text { Sociais }\end{array}$ \\
\hline 22 & Backtweets & Sim & Não & Sim & Não & Análise De Sentimentos No Twitter \\
\hline 23 & Social Bakers & Sim & Não & Sim & Não & $\begin{array}{l}\text { Compara Os Sentimentos De } 2 \\
\text { Concorrentes No Facebook }\end{array}$ \\
\hline
\end{tabular}

A autora: 2016

\subsubsection{A ferramenta Tagul}

A principal vantagem está na possibilidade de gerar a nuvem de palavras em cima de formas personalizadas. Mas as funcionalidades são diversas. É possível criar uma nuvem a partir de um endereço web ou de um texto e ainda eliminar ou adicionar palavras desejadas. A configuração estética da nuvem está cheia de opções: tipo de letra, cores, fundo, forma gráfica, etc.

75 Revista Competitividade e Sustentabilidade - ComSus, Paraná, v. 3, n. 2, p. 67-81, Jul /Dez. 2016. 
A utilização deste serviço requer registo de utilizador no site e o plug-in do Adobe Flash instalado no computador.

\subsubsection{Ferramenta $\mathrm{R}$}

O R é uma ferramenta para o desenvolvimento de sistemas de apoio a decisão e análise de dados tal como a execução de tarefas mais complexas que envolvam programação. Uma das suas principais características é o seu carácter gratuito, estando disponível em diversas plataformas (Windows, Linux, MacOS). Tem origem na linguagem S, desenvolvida nos laboratórios da AT\&T Bell por Rick Becker, John Chambers e Allan Walkins. Esta é uma ferramenta de código é aberto (Open Source), ou seja, é susceptível de ser alterado pelo utilizador quer para modificação das funcionalidades existentes quer para o desenvolvimento de novas funcionalidades. É uma ferramenta bastante poderosa com uma programação por objetos e um conjunto bastante vasto de pacotes que acrescentam bastantes potencialidades.

\section{PROCEDIMENTOS METODOLÓGICOS}

Para um melhor entendimento acerca do tema abordado, foram feitas inicialmente consultas a bibliografias especializadas, artigos de internet, e órgãos como a FBAJ (Federação Internacional de Albergues da Juventude) e a HI Hostelling International a fim de levantar informações sobre o destino Brasil, mais precisamente Santa Catarina, o produto hoteleiro e seus meios de classificação, o turismo alternativo e os Albergues da Juventude. Paralelamente, foi realizada uma pesquisa exploratória a fim de catalogar os Albergues da Juventude existentes em todo o Estado.

Sendo assim a busca se concentrou em 5 sites: Tripadvisor, Hostelbookers, Hostelworld, Trivago e Travelholics e nas páginas do Facebook destes albergues.

Com isso foram encontrados 110 albergues/ hostels localizados na região de Santa Catarina (Região da Praia do Rosa e Garopaba, Grande Florianópolis, Blumenau, Joinville, São Francisco do Sul, Bombinhas e Balneário Camboriú).

Para efetuar uma análise foi utilizado o site de maior relevância que foi o Tripadvisor, sendo assim, foram copiados os principais comentários referentes aos albergues encontrados e colocados nas ferramentas Tagul e R para efetuar uma análise.

Com isso foram levantadas várias palavras, retiradas as irrelevantes como conjunções e artigos, e após isto feita uma nuvem de palavras com os principais dados, levando em considerações diferentes tipos de ênfases e análises. 


\section{ANÁLISE DOS DADOS E INTERPRETAÇÃO DOS RESULTADOS}

Foram coletados do site Tripadvisor 2.976 comentários, referentes aos 110 albergues analisados e utilizados estes dados para realizar algumas análises.

Primeiramente foi feita uma nuvem de palavras dando destaque aos albergues que obtiveram um maior número de comentários, sem diferenciar se eram positivos ou negativos, os maiores destaques foram: Sunset Backpackers, Barra Beach Club, Hostel Energia da Lagoa, Lagoa Hostel, Sunset Strip Eco Hostel.

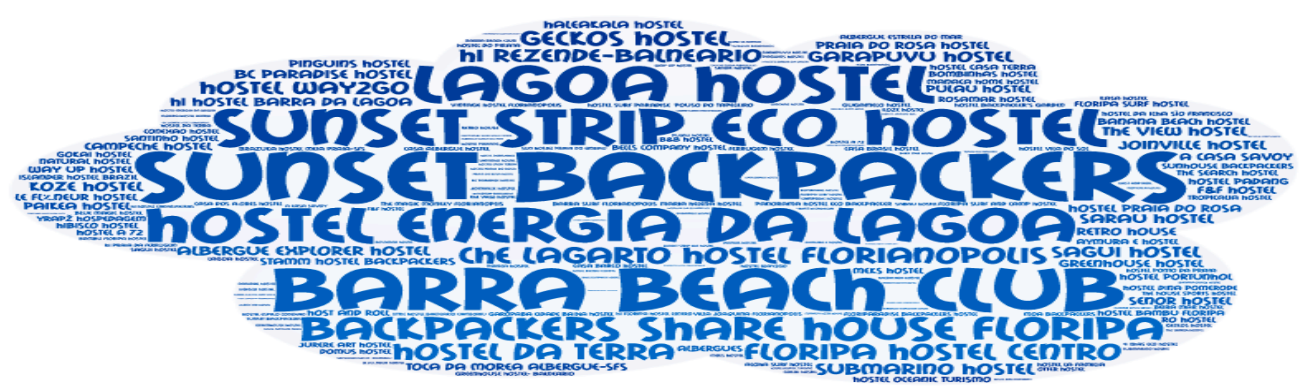

Fonte: Tagul. 2016.

A segunda opção foi efetuar uma busca no Tagul, colocando apenas como dados: Hostels em Santa Catarina, diretamente da página do Tripadvisor, verificando que as informações geradas são muito genéricas e não dizem nada a respeito dos comentários dos albergues. Palavras como: Florianópolis, hotéis, preços, avaliações e quartos, que dão apenas uma ideia do que pode ser abordado em um comentário no site.

77 Revista Competitividade e Sustentabilidade - ComSus, Paraná, v. 3, n. 2, p. 67-81, Jul /Dez. 2016. 


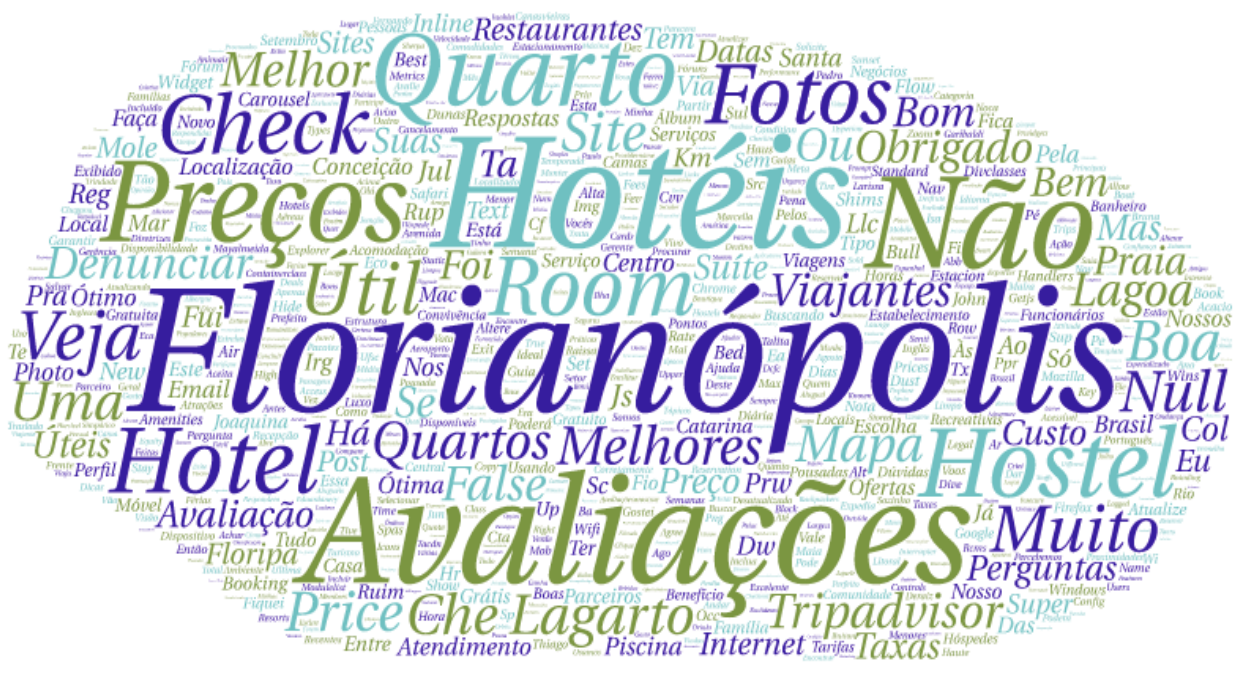

Fonte: Tagul. 2016.

Depois disso foram copiadas as informações dos comentários referentes a todos os albergues de Santa Catarina e importados para a ferramenta Tagul, desta forma as informações foram muito mais precisas e detalhadas. Através da formação da nuvem de palavras é possível perceber que a maior parte dos comentários feitos pelos hóspedes são positivos, tendo como palavras que foram repetidas mais vezes: Hostel, bom, ótimo, super, boa, praia, limpo, ótima, melhor, excelente e bem.

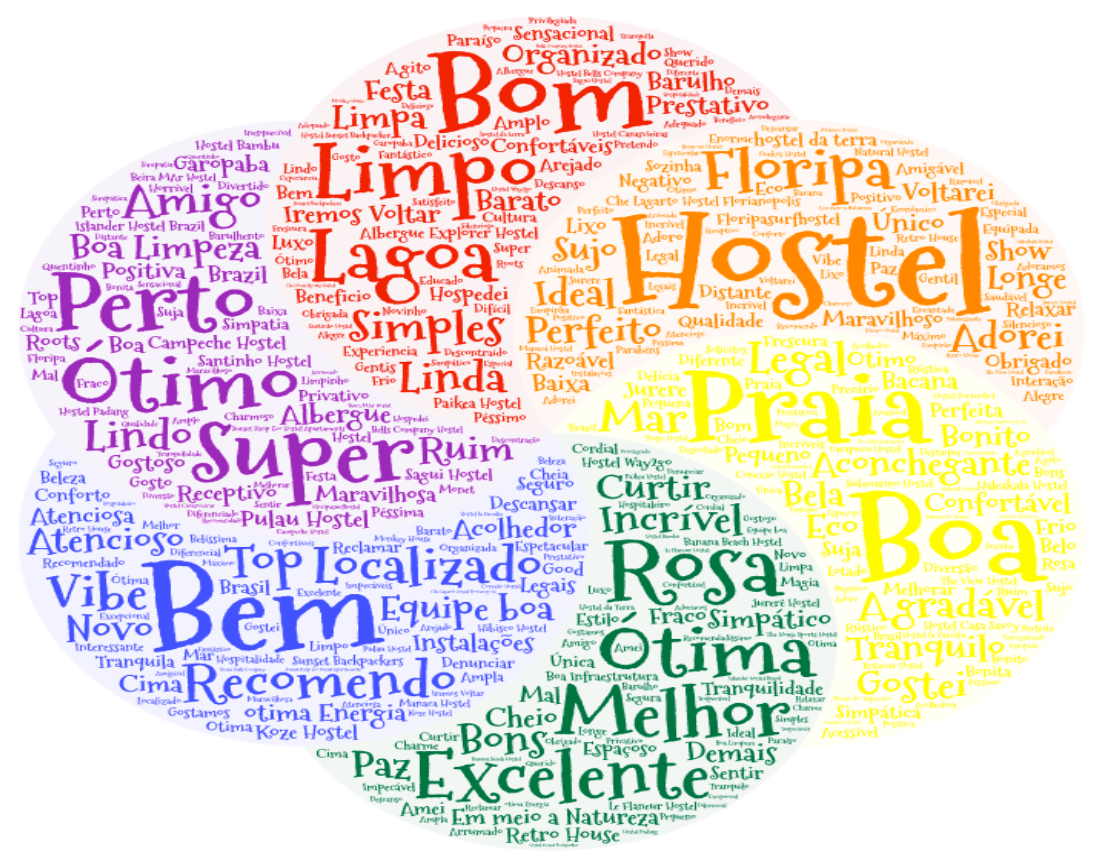

Fonte: Tagul. 2016.

78 Revista Competitividade e Sustentabilidade - ComSus, Paraná, v. 3, n. 2, p. 67-81, Jul /Dez. 2016. 


\section{ANÁLISE DE SENTIMENTOS DOS ALBERGUES DE SANTA CATARINA ATRAVÉS DOS COMENTÁRIOS NO SITE...}

Também foi realizada a análise de sentimentos com a ferramenta $R$, que gerou uma nuvem de palavras, onde também foi possível notar que a maioria dos comentários gerados foram positivos como: excelente, bom, melhor, ótimo, agradável, acolhedor, aconchegante. Sendo uma quantidade bem menor de termos negativos como: sujo e péssimo.

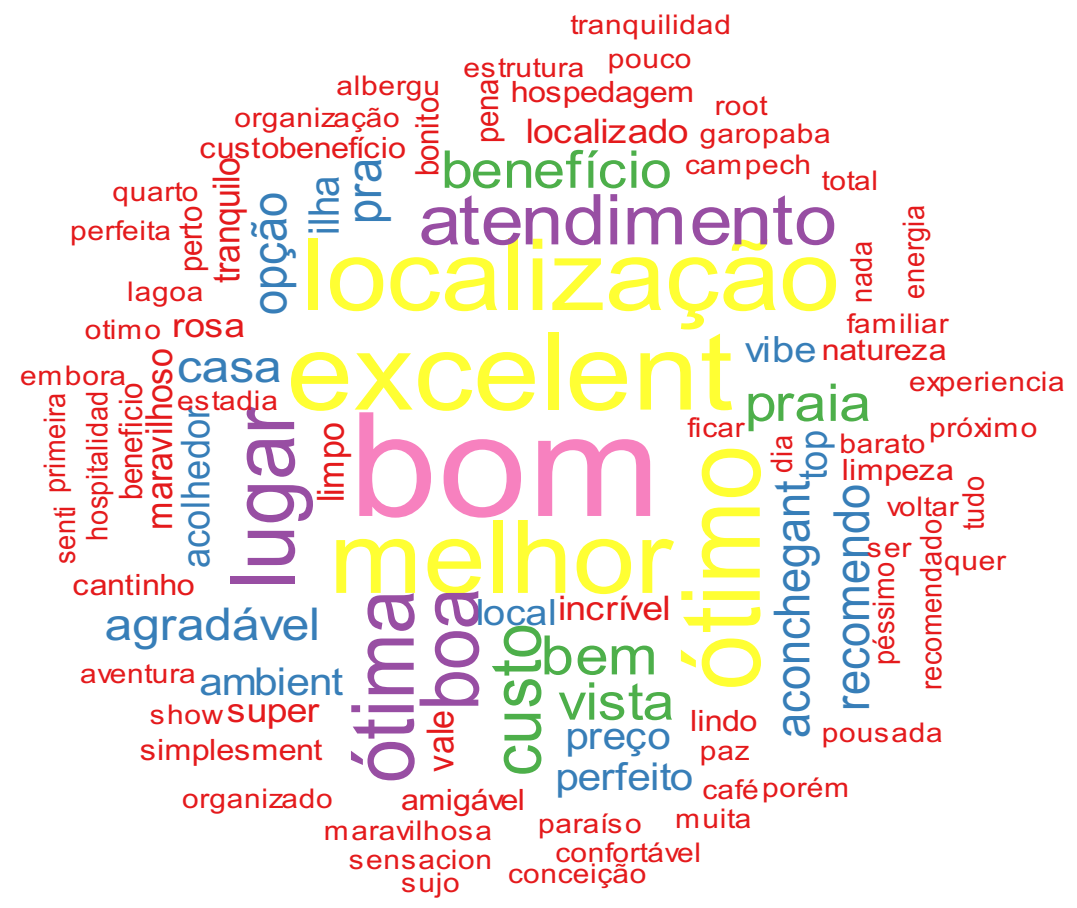

Fonte: Sistema R.2016

Pelo mesmo sistema R é possível elaborar um gráfico no qual se verificam quais foram as 15 palavras mais repetidas na mineração de dados, e mais uma vez é possível verificar que os sentimentos positivos são os mais utilizados.

79 Revista Competitividade e Sustentabilidade - ComSus, Paraná, v. 3, n. 2, p. 67-81, Jul /Dez. 2016. 


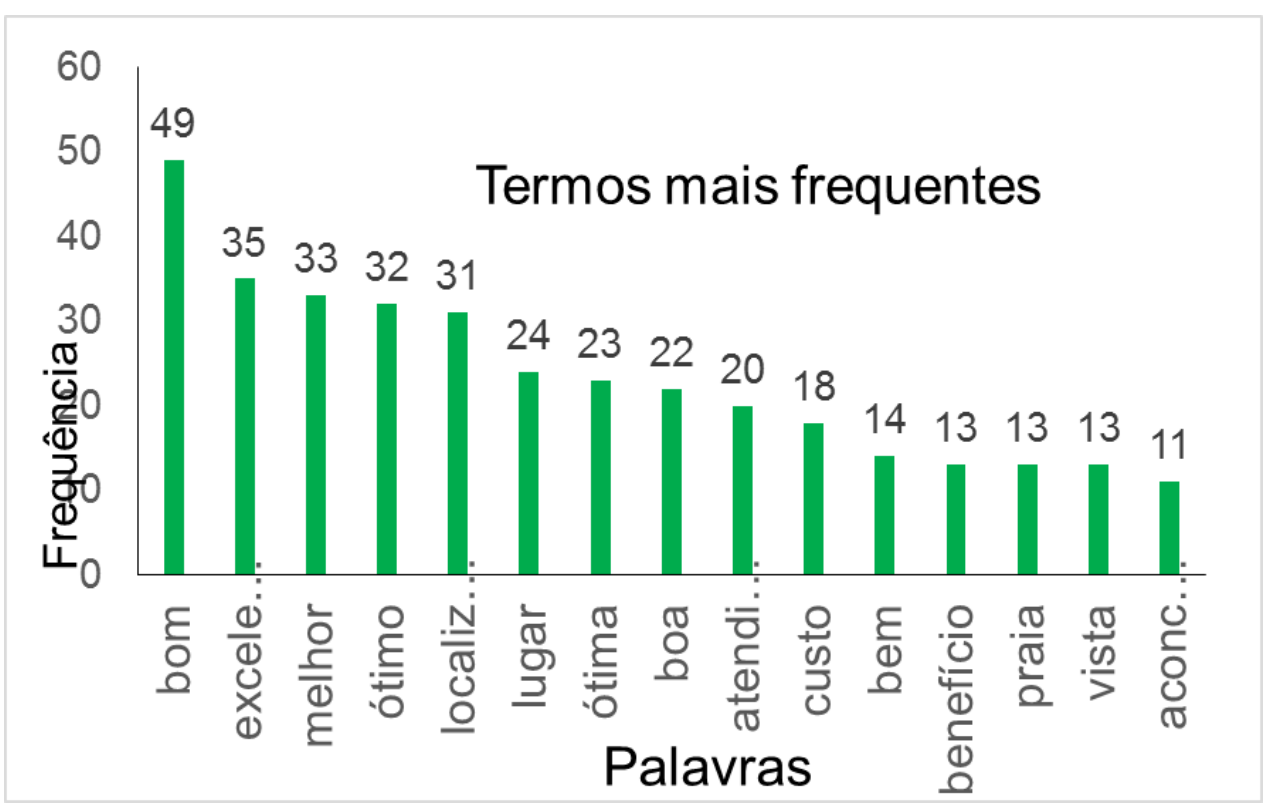

Fonte: Sistema R. 2016.

\section{CONSIDERAÇÕES FINAIS}

Conforme foi citado durante a pesquisa foi possível perceber que as redes sociais possuem uma grande influência na reputação dos albergues, uma vez que não existe nenhum tipo de regulamentação ou de classificação em estrelas para os albergues/hostels e é principalmente através delas que os viajantes conseguem buscar opiniões a respeito de um determinado local que ainda não conhecem.

Mas de uma forma geral é possível dizer que os estabelecimentos possuem na sua maioria, uma opinião coesa a respeito dos comentários, tendo pequenas discrepâncias, que são decorrentes de algum problema pessoal que tenha ocorrido durante a viagem.

Ao efetuar uma comparação entre a ferramenta Tagul e o $\mathrm{R}$, foi possível constatar que a ferramenta Tagul, possui mais recursos disponíveis para que se façam diversos tipos de nuvens de palavras, tanto referente à parte gráfica quanto referente aos recursos online e de planilha. Além de ser uma ferramenta gratuita e online. A ferramenta R é um pouco mais complexa e demanda uma experiência um pouco maior de quem a utiliza.

Entretanto mesmo com a utilização de duas ferramentas diferentes foi possível constatar que a maioria dos comentários foi positiva. Isso nos indica que se um elevado número de pessoas possui uma opinião semelhante sobre um mesmo local, podemos considerar essa opinião como verdadeira e uma ótima referência para viajantes futuros. 


\section{REFERÊNCIAS}

Andrade, José Vicente de. Turismo: Fundamentos e Dimensões, $8^{\mathrm{a}}$ ed. São Paulo: Ática, 2004.

Becker, Karin; Tumitan, Diego. Introdução à mineração de opiniões: conceitos, aplicações e desafios. In: SIMPÓSIO BRASILEIRO DE BANCO DE DADOS - SBBD, 28, 2013, Recife. Anais... [s.i.]: Sbc, 2013. p. 1 - 26.

Beni, Mário C. Análise Estrutural do Turismo. 8. ed. São Paulo: Editora SENAC, 2003.

Castelli, Geraldo. Administração Hoteleira. 9. ed. São Paulo: EDUCS, 2001. 594 p.

Dias, C.M.M (Org). Hospitalidade: reflexões e perspectivas. São Paulo: Manole, 2002.

Hi. Hostelling International. Quem Somos. Disponível em: http://www.hihostelbrasil.com.br/ Acesso em: 05 jun. 2016.

Hostelbookers. Disponível em: http://pt.hostelbookers.com/. Acesso em: 12 mai. 2016.

Hostelworld. Disponível em: http://www.hostelworld.com/. Acesso em: 15 maio. 2016.

Liu, Bing. Sentiment analysis and opinion mining: Synthesis Lectures on Human Language Technologies. [s.i.]: Morgan \& Claypool Publishers, 2012. 168 p. Disponível em: https://www.cs.uic.edu/ liub/FBS/SentimentAnalysis-and-OpinionMining.pdf .Acesso em: 12 jul. 2016.

Omt, Organização Mundial Do Turismo. Introdução ao turismo. São Paulo: Roca, 2001.

Marketing De Conteúdo. Disponível em:

http://marketingdeconteudo.com/ferramentas-gratuitas-para-monitoramento-de-redes-sociais/. Acesso em 12 de outubro de 2016.

Monard, M. C. \& J. A. Baranauskas (2003). Conceitos sobre aprendizado de maquina. In S. O. Rezende (Ed.), Sistemas Inteligentes: Fundamentos e Aplicações, pp. 89- 114. Manole

Sistema R. Disponível em: http://www.r-project.org/ Acesso em 13 de outubro de 2016.

Tagul. Disponível em https://tagul.com/cloud/1. Acesso em 12 de outubro de 2016.

Tarapanoff, Kira. Inteligência, informação e conhecimento em corporações. Instituto Brasileiro de Informação em Ciência e Tecnologia (IBICT), 2006.

Travelholics. Disponível em: http://www.travelaholics.com.br.Acesso em: 18 maio. 2016.

Tripadvisor. Disponível em: https://www.tripadvisor.com.br/ Acesso em: 15 de outubro 2016. Trivago. Disponível em: http://www.trivago.com.br/. Acesso em: 10 maio. 2016.

\section{NOTAS}

1 Programa de Pós-Graduação de Ciência e Gestão da Informação - UFPR- E-mail: lucianaaraujo@utfpr.edu.br

81 Revista Competitividade e Sustentabilidade - ComSus, Paraná, v. 3, n. 2, p. 67-81, Jul /Dez. 2016. 